\title{
Hepatic MRI for GE Scanners
}

MRI techniques continue to evolve rapidly, but the basic components of a liver examination, and the clinical role of each component, have changed little. The three most important considerations for choosing techniques for imaging the liver continue to be contrast, motion artifact, and coverage. In particular, the approach to motion has evolved from averaging to motion compensation to suspended respiration and/or subsecond imaging.

This unit is written as an alternate to UNIT A15.1, which bases recommendations on experience using Siemens MRI equipment. The authors' experience has been acquired primarily using General Electric equipment. However, these basic approaches to hepatic MR imaging are quite similar, with most details relating to minor vendor-specific differences.

\section{LIVER IMAGING}

Whenever possible, it is best to image the entire liver during a single breath-hold sequence. The advantages of single breath-hold coverage include simplicity and avoidance of misregistration. Single breath-hold imaging being essential for dynamic multiphasic imaging must be emphasized; the crucial arterial phase can only be acquired during a brief interval of $\sim 20 \mathrm{sec}$. Whenever possible, patients should be triaged to MRI machines that have this capability. The abdomen ranks along with the heart and vascular system as body parts demanding the most up-to-date MRI hardware and software for successful use.

Several years ago, successful use of MRI for imaging the liver usually involved close involvement of the senior MR radiologist with each scanning procedure. During the $\geq 60$ min that these exams often required, the radiologist would spend much of his/her time behind the scanner discussing the details of next pulse sequence with the technologist during the 5 to 10 min that would elapse during each sequence acquisition. Cases would be read between exams.

Times have changed. Markedly increased caseloads and decreased reimbursements no longer permit this luxury. Hepatic MRI can only become a viable procedure for routine use if the execution of protocols can be trusted to a trained technologist, freeing the radiologist to read cases and interact with referring clinicians. Fortunately, hardware and software improvements now permit this, and the comprehensive hepatic MRI protocol described here should not require $>30$ min of MRI scanner time. Continued improvements in the scanner interface will allow these exams to be completed in $\sim 10 \mathrm{~min}$; actual image acquisition is $<5 \mathrm{~min}$, with all images acquired during breath holding. These sequences require the patient to be able to suspend respiration for $\sim 25 \mathrm{sec}$. It is imperative that there is clear communication between the technologist and the patient throughout the exam. If patients are unable to cooperate with this, there are alternative pulse sequences that can be used to yield images of acceptable quality.

Table A15.2.1 lists the hardware necessary to perform the procedure, along with appropriate parameters.

NOTE: Be sure that technologists and nurses have immediate access to any emergency equipment that may be relevant to a given study, or that may be needed for a particular patient, such as crash carts or oxygen.

Contributed by Donald G. Mitchell, Peter Natale, and George Holland

Current Protocols in Magnetic Resonance Imaging (2002) A15.2.1-A15.2.14

Copyright $\odot 2002$ by John Wiley \& Sons, Inc.
BASIC

PROTOCOL

Liver

A15.2.1

Supplement 4 
Table A15.2.1 Equipment Specifications Needed to Perform Imaging Sequences

\begin{tabular}{ll}
\hline Coil type & Torso phased array coil \\
Manufacturer and system type & GE Signa \\
Field strength & $1.5 \mathrm{~T}$ \\
Gradient strength & $25 \mathrm{mT} / \mathrm{m}$ (or whatever the system permits) \\
Knee cushion & Yes \\
Pulse oximeter & If patient requires sedation \\
Power injector & Yes, if available \\
Normal saline & Yes \\
35-in. extension tubing & Yes \\
Use of contrast agents & Yes \\
\hline
\end{tabular}

\section{Materials}

Normal saline $(0.9 \% \mathrm{NaCl})$, sterile, $100 \mathrm{ml}$ minimum

Extravascular contrast agent (e.g., Magnevist, Omniscan, or Prohance), $20 \mathrm{ml}$ for most patients

22-G angiocatheter

110 -in. extension tubing

\section{Set up patient and equipment}

1. Interview (screen) the patient to assess for contraindications such as cardiac pacemaker, implanted mechanical devices, and/or ferromagnetic materials. Also, determine if the patient will need sedation medication necessitating the need for appropriate monitoring equipment.

Generally, standard screening forms (APPENDIX 1) are used for all patients screened in a magnetic resonance system.

The presence of ferromagnetic materials may be a health hazard to the patient while in the magnetic field and/or adversely affect image quality. To determine the safety of scanning such ferromagnetic materials see Shellock and Kanal (1996).

The presence of ferromagnetic materials in the globe of the eye is contraindicated for MRI. Patients with prior metal exposure to the eye should have plain X-rays of the orbital area to ensure that all metal has been removed prior to placing them in the magnetic field.

Patients may be accompanied into the magnet room by a friend or family member, who can sit in the room during the scan and comfort the patient as needed. This companion must be screened as well to ensure the absence of loose metal objects on the body or clothing.

2. Request the patient to change into a gown to eliminate any metal that might be found in clothing. Ask the patient to remove all personal effects such as jewelry, hearing aids, glasses, etc., prior to entering the MRI scan room.

\section{All personal belongings should be secured during the examination.}

3. Explain the procedure to the patient and record relevant clinical history. Ensure that the patient understands what is expected and ask if they have any questions; answer appropriately. If this is a research protocol, have the patient sign any necessary consent forms.

4. Obtain intravenous (i.v.) access utilizing a 22-G angiocatheter and attach to saline filled extension tubing (110-in.), with saline set to slow infusion by gravity drip. Secure position of angiocatheter with sterile, clear occlusive dressing. 
Obtaining i.v. access prior to entering the scan room will promote patient throughput and eliminate "dead" time of starting the i.v. while the patient is on the exam table. Follow power injector manufacturer guidelines with regard to appropriate needle gauge/angiocatheter to be used; this will depend on the chosen injection/flow rates.

If one does not have access to a power injector, one will still be able to perform dynamic liver imaging. In this case, draw-up the contrast agent in one 20-ml syringe and saline in two others to flush the timing and examination boluses. Alternatively, larger syringes can be used, drawing 20-ml of contrast agent into one and 40-ml of saline into the other. To eliminate the need of attaching and detaching syringes when switching from contrast agent to flushing the saline, incorporate the use of a 3-way stopcock or Y-tubing.

5. Set up the power injector as specified by the manufacturer.

A minimum of $40 \mathrm{ml}$ normal saline should be drawn-up to ensure sufficient saline is available to keep the vein open (KVO) throughout the exam. There is no need to use a double dose for hepatic MRI. While the standard on-label dose is $0.1 \mathrm{mmol} / \mathrm{kg}$, this can safely be rounded up to the nearest bottle size, or a dose of $20 \mathrm{ml}$ can be used routinely.

6. Escort the patient to the MR examination room and ask them to lie down accordingly with respect to the exam to be performed. Connect the extension tubing secured to the syringe, to the power injector extension tubing. Review the following items with the patient:

a. Provide earplugs or headphones to the patient to minimize the loud knocking noise that will be produced by the gradients but ensure them that they will still be able to hear you.

b. Provide the patient with a safety squeeze-bulb and demonstrate how it works; explain to the patient when to use the squeeze-bulb (i.e., if they need assistance during the exam).

c. Explain to the patient that you will be talking to them between imaging sequences which will be when the loud knocking noise stops. Additionally, review breathholding instructions with the patient.

d. Explain to the patient that it is imperative that they remain motionless during the loud knocking noise to ensure good results; also explain that they should not reposition their body between imaging sequences.

e. Nevertheless, the patient may call out at any time if he or she feels it necessary.

f. Provide the patient with an approximate time that the examination will take.

g. Attach the power injector to the i.v., and set it to keep the line open.

7. Place the torso phased array coil on the scanning table. Secure the torso phased array coil to the patient, centering at the costal margin in most patients. Usually, straps are provided by the manufacturer that are directly attached to the coil. Place a foam pad between the patient's abdomen and the coil to reduce near-field artifacts. Place a support under the patient's knees to enhance patient comfort.

8. Place nasal canula for oxygen 2 liters, if available, to aid in suspended respiration. Hyperventilation before breathholding is helpful. Most helpful is administration of oxygen during the examination (Marks et al., 1997).

9. Using the laser light, center the patient's upper abdomen to the coil.

10. Advance the patient table to isocenter.

Once this step has been performed, so long as the patient does not move on the table, the table itself can be moved and then replaced in the same position as before without jeopardizing the positioning of one scan relative to another. 
11. Program the power injector for a contrast agent and saline injection rate of $2 \mathrm{ml} / \mathrm{sec}$. Total volume of saline following contrast agent injection should be programmed for $20 \mathrm{ml}$. The scan delay in healthy young patients averages $18 \mathrm{sec}$ (contrast agent is injected, scan is initiated $18 \mathrm{sec}$ after the contrast agent and saline are delivered), but this is highly variable in the elderly or those with cardiovascular disease.

Although Fisher, Semelka, and Wilber (UNITA15.1) recommend the routine use on an 18-sec delay, since this is a good estimate for most healthy individuals, the authors strongly recommend determination of the optimum delay in each patient. This can either be done with the automated bolus detection (SMARTPREP, General Electric) feature, or by use of a $2 \mathrm{ml}$ timing bolus sequence, as per Earls et al. (1997) and described later in this unit. Both of these methods can be adapted to either hand or power injection.

12. Arm the power injector and keep the vein open. IMPORTANT: Do not inject the contrast agent.

\section{Sequence 1: Coronal single shot fast spin echo (SSFSE)}

Although some technologists precede this with a three-plane orthogonal scout sequence, the authors find that this can be safely omitted by most experienced technologists. The coronal single shot fast spin echo (SSFSE) sequence is nearly always of high quality and high information content (Fig. A15.2.1). These can be acquired using a $320-$ to $360-\mathrm{mm}^{2}$ field of view (FOV) and 5- to 8-mm image slices. Using thicker image slices will reduce the number of slices and save imaging time. Using a large FOV allows additional imaging of the abdomen and much of the lower chest and upper pelvis in a single breathhold, and provides added assurance against wrap-around artifact. Alternatively, a smaller FOV and thinner image slices $(5 \mathrm{~mm})$ can be acquired in two or more breathholds to provide greater detail of biliary and pancreatic ducts. Each breath-hold should cover one slab (with a certain number of slices) such that the scan time is $\sim 20 \mathrm{sec}$. Then move the region of interest to a new slab. Slabs should be overlapped, not interleaved.

13. Instruct the patient to remain motionless, and to take a deep breath in and blow it out two times, then hold it. Run the sequence according to the parameters, except with 14 number of slices, listed in Table A15.2.2. Instruct the patient to breathe and hold their breath, run the sequence to cover the remaining 14 slices.

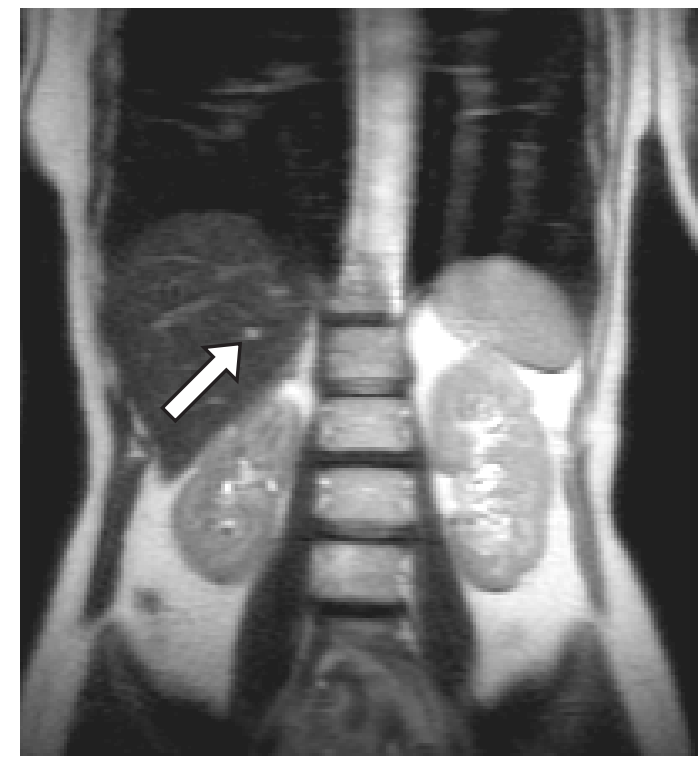

Hepatic MRI for GE Scanners

A15.2.4 
Table A15.2.2 Imaging Parameters for Sequence 1 (Single Shot Fast Spin Echo)

\begin{tabular}{ll}
\hline Patient position & Supine \\
Scan type & Single shot fast spin echo \\
Imaging plane (orientation) & Coronal \\
Variable bandwidth & Yes \\
Pulse sequence database (PSD) & SSFSE \\
Central slice or volume center & Center to xyphoid \\
Echo time $\left(T_{\mathrm{E}}\right)$ & $100 \mathrm{msec}(\mathrm{effective})$ \\
Receiver bandwidth $(\mathrm{RBW})$ & $\pm 31 \mathrm{kHz}$ \\
Repeat time $\left(T_{\mathrm{R}}\right)$ & Infinite \\
Flip angle $(\mathrm{FA})$ & $90^{\circ}$ \\
Fields of view $(\mathrm{FOV}, \mathrm{FOV}$ & \\
Resolution $(\Delta x, \Delta y)$ & $360 \mathrm{~mm}, 360 \mathrm{~mm}$ \\
Number of data points collected $\left(N_{\mathrm{x}}, N_{\mathrm{y}}\right)$ & $1.41 \mathrm{~mm}, 1.92 \mathrm{~mm}$ \\
Display matrix $\left(D_{\mathrm{x}}, D_{\mathrm{y}}\right)$ & $256, \sim 100$ \\
Slice thickness $(\Delta z)$ & 256,256 \\
Number of slices & $5 \mathrm{~mm}$ \\
Slice gap & 28 \\
Number of excitations $(\mathrm{NEX})$ & 0 \\
Swap read and phase encoding & $0.5(\mathrm{half}$ Fourier) \\
Slice location & No \\
Saturation pulses & A70-P70 \\
Scan time & None \\
\hline
\end{tabular}

Obtaining images during suspended respiration will minimize misregistration and image blurring. However, it is important to be aware that the absence of visible motion artifact does not indicate that the patient successfully suspended respiration; the multislice gradient echo images must be checked for this.

Although some patients may hold their breath longer at full inspiration, more consistent location and absence of motion are achieved if respiration is suspended at relaxed expiration.

Observe the respiratory waveform to determine if the patient is complying with breath holding instructions. Remember that poor compliance will not degrade these images because of their relative lack of motion sensitivity.

14. Confirm positioning of the torso coil by making certain that all parts of the liver are included within the sensitive volume of the coil. Reposition the patient and repeat the sequence, if necessary.

Sequence 2: Heavily $T_{2}$-weighted $\left(T_{E}=180 \mathrm{msec}\right)$ transverse single shot FSE

15. Using the coronal images, position slices to ensure that the entire liver is covered on transverse images (Fig. A15.2.2). Ask the patient to hold his or her breath and run the sequence according to the parameters listed in Table A15.2.3.

\section{Sequence 3: Transverse gradient echo in-phase and out-of-phase}

If possible, these should be obtained as a dual-echo sequence, with $T_{\mathrm{E}} \mathrm{s}$ of $\sim 2.3 \mathrm{msec}$ (out-of-phase) and $4.6 \mathrm{msec}$ (in-phase; Fig. A15.2.3). If this is not available, separate in-phase and out-of-phase acquisitions will be necessary. Although the $T_{\mathrm{E}} \mathrm{s}$ mentioned above are ideal, software may be configured to yield slightly different $T_{\mathrm{E}} \mathrm{s}$, with acceptable results. 


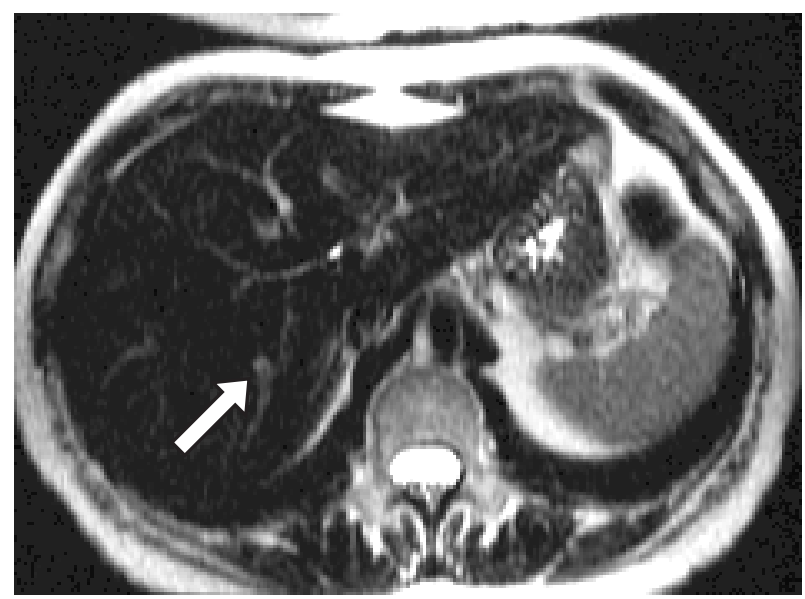

Figure A15.2.2 Heavily $T_{2}$-weighted transverse single shot FSE image, with effective $T_{E}=180$ msec. Arrow indicates the small cavernous hemangioma, with signal intensity intermediate between that of spleen and cerebrospinal fluid.

Table A15.2.3 Imaging Parameters for Sequence 2 (Single Shot Fast Spin Echo)

\begin{tabular}{ll}
\hline Patient position & Supine \\
Scan type & Single shot fast spin echo \\
Imaging plane (orientation) & Transverse \\
Variable bandwidth & Yes \\
Pulse sequence database (PSD) & SSFSE \\
Central slice or volume center & Center to liver \\
Echo time $\left(T_{\mathrm{E}}\right)$ & $180 \mathrm{msec}(\mathrm{effective})$ \\
Receiver bandwidth $(\mathrm{RBW})$ & $\pm 31 \mathrm{kHz}$ \\
Repeat time $\left(T_{\mathrm{R}}\right)$ & Infinite \\
Flip angle $(\mathrm{FA})$ & $90^{\circ}$ \\
Fields of view $(\mathrm{FOV}, \mathrm{FOV}$ & $320 \mathrm{~mm}, 240 \mathrm{~mm}$ \\
Resolution $(\Delta x, \Delta y)$ & $1.25 \mathrm{~mm}, 2.00 \mathrm{~mm}$ \\
Number of data points collected $\left(N_{\mathrm{x}}, N_{\mathrm{y}}\right)$ & $256, \sim 120$ \\
Display matrix $\left(D_{\mathrm{x}}, D_{\mathrm{y}}\right)$ & 256,256 \\
Slice thickness $(\Delta z)$ & $5 \mathrm{~mm}$ \\
Number of slices & 24 \\
Slice gap & 0 \\
Number of excitations $(\mathrm{NEX})$ & 0.5 (half Fourier) \\
Swap read and phase encoding & No \\
Slice location & Entire liver \\
Saturation pulses & Superior and inferior \\
Scan time & $24 \mathrm{sec}$ \\
&
\end{tabular}

Hepatic MRI for GE Scanners

A15.2.6

16. Position slices to cover entire liver, as for sequence 2, adjusting levels if necessary. Ask the patient to hold his or her breath and run the sequence according to the parameters listed in Table A15.2.4.

17. Evaluate for image quality.

This is the first motion-sensitive scan, and will therefore be the first to be degraded if breath holding is not successful. If there is substantial motion artifact, remind the patient about the importance of breath holding, and repeat the acquisition. Consider reducing coverage or acquisition matrix, or increasing image thickness, to reduce acquisition time if necessary. 


\section{Sequence 4: Timing bolus sequence (if automated bolus detection is not used)}

If automated bolus detection (e.g., SMARTPREP) is not used, the delay between injection and scanning should be determined by injecting a $2-\mathrm{ml}$ test bolus, followed by a $20-\mathrm{ml}$ flush of saline (Earls et al., 1997). The size of the syringe used should be the same as that used for injecting the bolus of gadolinium chelate, and the speed of injection should be as identical as possible. The pulse sequence used for the test bolus on GE equipment should be a single 20 - $\mathrm{mm}$ sagittal slice short $T_{\mathrm{R}}$ gradient echo sequence (60 multi-phases),

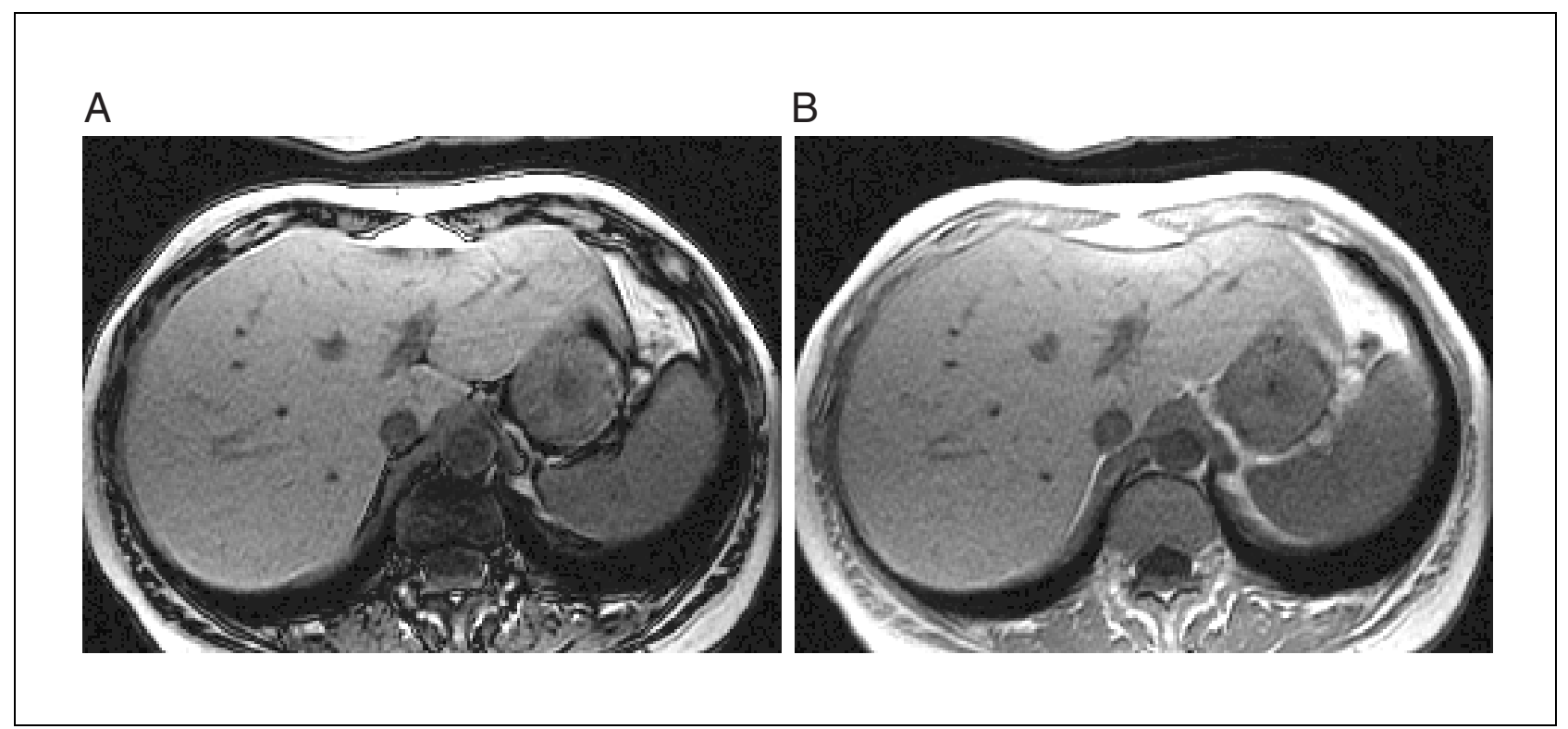

Figure A15.2.3 Spoiled gradient echo images: out-of-phase, with $T_{E}=2.3 \mathrm{msec}(\mathbf{A})$ and in-phase, with $T_{E}=4.6 \mathrm{msec}(\mathbf{B})$.

Table A15.2.4 Imaging Parameters for Sequence 3 Dual (Gradient Echo)

\begin{tabular}{|c|c|}
\hline Patient position & Supine \\
\hline Scan type & 2-D double gradient echo \\
\hline Imaging plane (orientation) & Transverse \\
\hline Variable bandwidth & Yes \\
\hline Pulse sequence database (PSD) & FGRE-dual \\
\hline Central slice or volume center & Center to liver \\
\hline Echo time $\left(T_{\mathrm{E}}\right)$ & $2.3 \mathrm{msec}$ and $4.6 \mathrm{msec}$ \\
\hline Receiver bandwidth (RBW) & $\pm 62 \mathrm{kHz}$ \\
\hline Repeat time $\left(T_{\mathrm{R}}\right)$ & $200 \mathrm{msec}$ \\
\hline Flip angle (FA) & $90^{\circ}$ \\
\hline Fields of view $\left(\mathrm{FOV}_{\mathrm{x}}, \mathrm{FOV}_{\mathrm{y}}\right)$ & $320 \mathrm{~mm}, 240 \mathrm{~mm}$ \\
\hline Resolution $(\Delta x, \Delta y)$ & $1.25 \mathrm{~mm}, 3.00 \mathrm{~mm}$ \\
\hline Number of data points collected $\left(N_{\mathrm{x}}, N_{\mathrm{y}}\right)$ & $256, \sim 80$ \\
\hline Display matrix $\left(D_{\mathrm{x}}, D_{\mathrm{y}}\right)$ & 256,256 \\
\hline Slice thickness $(\Delta z)$ & $5 \mathrm{~mm}$ \\
\hline Number of slices & 24 \\
\hline Slice gap & 0 \\
\hline Number of excitations (NEX) & 0.5 (half Fourier) \\
\hline Swap read and phase encoding & No \\
\hline Slice location & Entire liver \\
\hline Saturation pulses & Superior, inferior \\
\hline Scan time & $24 \mathrm{sec}$ \\
\hline
\end{tabular}


Table A15.2.5 Imaging Parameters for Sequence 4 (Timing Bolus)

\begin{tabular}{ll}
\hline Patient position & Supine \\
Scan type & Gradient echo \\
Imaging plane (orientation) & Sagittal \\
Variable bandwidth & No \\
Pulse sequence database (PSD) & FGRE \\
Central slice or volume center & Upper abdominal aorta \\
Echo time $\left(T_{\mathrm{E}}\right)$ & Minimum $(\sim 2.1 \mathrm{msec})$ \\
Receiver bandwidth $(\mathrm{RBW})$ & $\pm 31 \mathrm{kHz}$ \\
Repeat time $\left(T_{\mathrm{R}}\right)$ & $7 \mathrm{msec}$ \\
Flip angle $(\mathrm{FA})$ & $70^{\circ}$ \\
Fields of view $(\mathrm{FOV}, \mathrm{x}, \mathrm{FOV}$ & $\mathrm{y})$ \\
Resolution $(\Delta x, \Delta y)$ & $320 \mathrm{~mm}, 240 \mathrm{~mm}$ \\
Number of data points collected $\left(N_{\mathrm{x}}, N_{\mathrm{y}}\right)$ & $1.25 \mathrm{~mm}, 2.50 \mathrm{~mm}$ \\
Display matrix $\left(D_{\mathrm{x}}, D_{\mathrm{y}}\right)$ & 256,96 \\
Slice thickness $(\Delta z)$ & 256,256 \\
Number of slices & $20 \mathrm{~mm}$ \\
Slice gap & 1 \\
Number of excitations $(\mathrm{NEX})$ & N/A \\
Swap read and phase encoding & 1 \\
Saturation pulses & No \\
Multi-phase & None \\
Scan time & 60 \\
\hline
\end{tabular}

with fields of view $320 \mathrm{~mm} \times 240 \mathrm{~mm}(3 / 4$ fields of view), centered over the upper abdominal aorta. Each phase should be obtained in $\sim 1 \mathrm{sec}$.

If the acquisition of the sequence begins when the flush begins, when the peak signal intensity in the mid-aorta is shown, the delay time is $5 \mathrm{sec}$ longer than the time it takes an injection of the contrast agent to travel from the i.v. tubing port to the abdominal aorta. This should correspond to the delay time between beginning of the major gadolinium bolus and the beginning of the acquisition. For example, if it takes $25 \mathrm{sec}$ for the gadolinium to travel from the i.v. tubing port to the mid abdominal aorta, breath-holding instructions should be given so that the actual pulse sequence begins $30 \mathrm{sec}$ following the beginning of the gadolinium injection.

18. Inject $2 \mathrm{ml}$ of gadolinium chelate, followed immediately by a $20 \mathrm{ml}$ flush of saline with an injection of $2 \mathrm{ml} / \mathrm{sec}$.

19. Run sequence 4 according to Table A15.2.5 at the beginning of the flush.

Breath holding is not necessary.

The aorta will have low signal intensity on all of these images, until the 2-ml bolus of gadolinium arrives, at which point there will be a noticeable increase in aortic signal intensity (Fig. A15.2.4). The time after the beginning of the acquisition is annotated in the upper left hand corner of the image as the delay time $\left(D_{T}\right)$ and can also be determined by noting the number of phases, and multiplying by 1 sec and then adding $5 \mathrm{sec}$.

\section{Sequence 5: Dynamic multi-phasic pre/post contrast 3-D short $T_{R}$ gradient echo}

Prescribe the volume to cover the liver. $T_{\mathrm{R}}$ and $T_{\mathrm{E}}$ should be as short as possible, and the flip angle should be $12^{\circ}$ to $15^{\circ}$. There will be visible contrast in the kidneys and collecting systems if a timing bolus was used, but there will be no significant effect on the liver. Obtain images (see Fig. A15.2.5) using the technique described above.

Hepatic MRI for GE Scanners

A15.2.8 
A

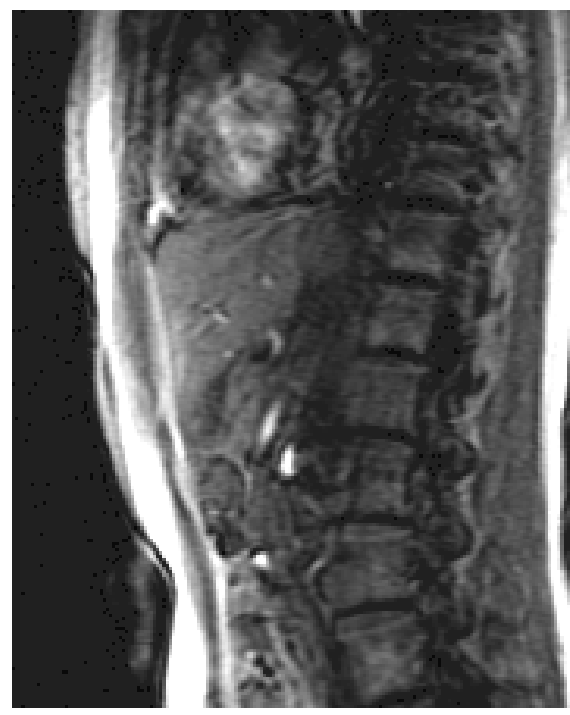

B

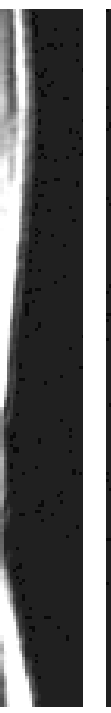

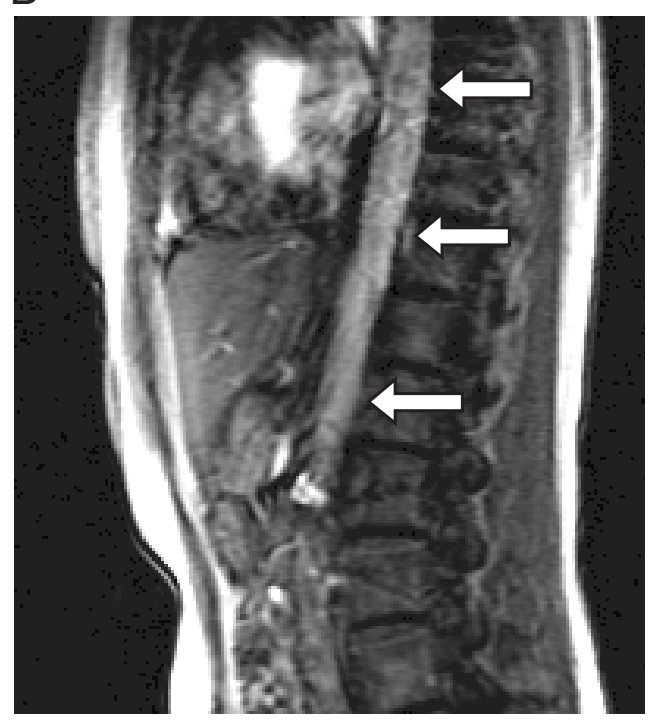

Figure A15.2.4 Selected images from sagittal timing bolus sequence, without (A) and during (B) aortic enhancement (arrows) from the 2-ml bolus of gadolinium chelate.

A

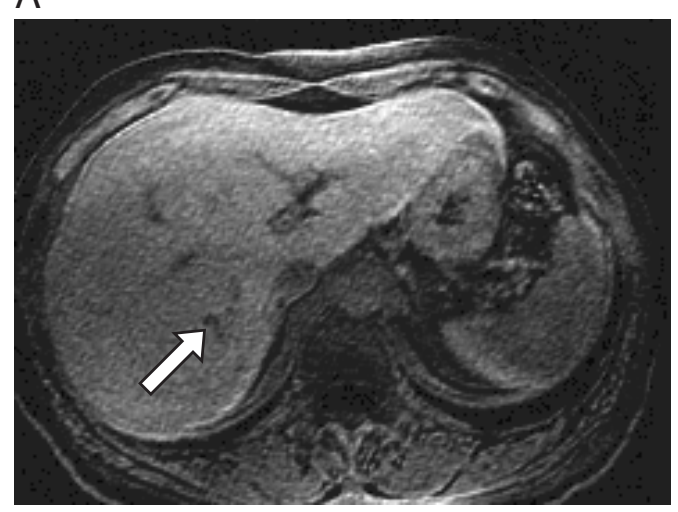

C

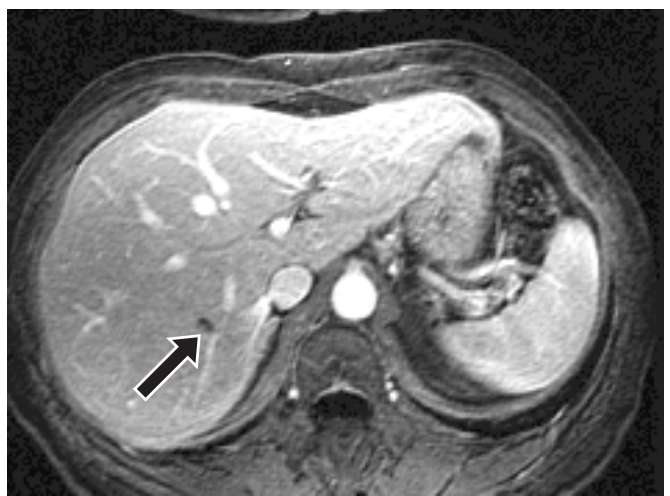

B

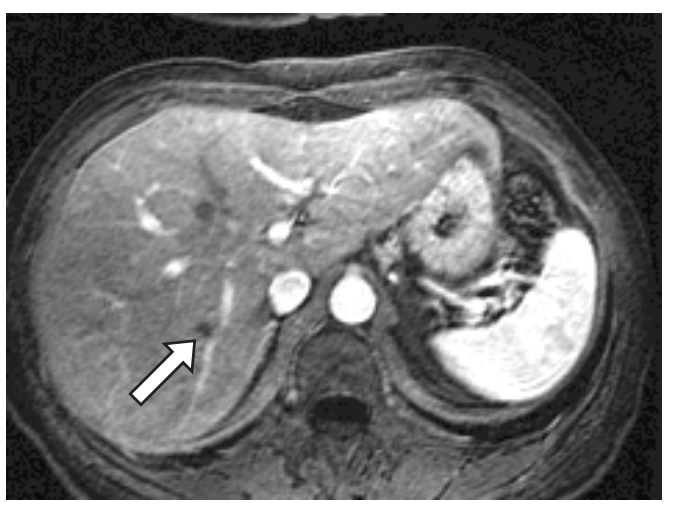

Figure A15.2.5 3-D fat-suppressed short $T_{\mathrm{R}}$ gradient echo images with $T_{\mathrm{R}} / T_{\mathrm{E}}=4 \mathrm{msec} / 1.3 \mathrm{msec}$, and flip angle $=12^{\circ}$, before (A), immediately after (B), and $45 \mathrm{sec}$ after (C) gadolinium chelate injection. Through Fourier interpolation, images are reconstructed every $2.5 \mathrm{~mm}$. Arrows indicate the cavernous hemangioma, which does not show enhancement in $\mathbf{A}$. 
Table A15.2.6 Imaging Parameters for Sequence 5 (Dynamic 3-D Gradient Echo)

\begin{tabular}{|c|c|}
\hline Patient position & Supine \\
\hline Scan type & 3-D gradient echo \\
\hline Imaging plane (orientation) & Transverse \\
\hline Variable bandwidth & Yes \\
\hline Pulse sequence database (PSD) & LUFGRE3D \\
\hline Central slice or volume center & Center to liver \\
\hline Echo time $\left(T_{\mathrm{E}}\right)$ & Minimum ( 1.5 msec) \\
\hline Receiver bandwidth (RBW) & $\pm 62 \mathrm{kHz}$ \\
\hline Repeat time $\left(T_{\mathrm{R}}\right)$ & $4 \mathrm{msec}$ \\
\hline Flip angle (FA) & $12^{\circ}-15^{\circ}$ \\
\hline Fields of view $\left(\mathrm{FOV}_{\mathrm{x}}, \mathrm{FOV}_{\mathrm{y}}\right)$ & $320 \mathrm{~mm}, 240 \mathrm{~mm}$ \\
\hline Resolution $(\Delta x, \Delta y)$ & $1.25 \mathrm{~mm}, 3.00 \mathrm{~mm}$ \\
\hline Number of data points collected $\left(N_{\mathrm{x}}, N_{\mathrm{y}}\right)$ & $256, \sim 80$ \\
\hline Display matrix $\left(D_{\mathrm{x}}, D_{\mathrm{y}}\right)$ & 256,256 \\
\hline Slice thickness $(\Delta z)$ & $5 \mathrm{~mm}$ \\
\hline Number of slices & 64 \\
\hline Slice gap & $-2.5 \mathrm{~mm}(50 \%$ overlap $)$ \\
\hline Number of excitations (NEX) & 0.5 (half Fourier) \\
\hline Swap read and phase encoding & No \\
\hline Slice location & Cover the entire liver \\
\hline ZIP2 & Yes \\
\hline Saturation pulses & None \\
\hline Fat suppression & $\begin{array}{l}\text { Yes, spectral inversion at lipids } \\
\text { (SPECIAL) }\end{array}$ \\
\hline Scan time & $24 \mathrm{sec}$ \\
\hline
\end{tabular}

20. Instruct the patient to hold his/her breath. Run the 3-D gradient echo sequence according to Table A15.2.6.

21. Explain to the patient that the contrast agent will now be injected and that they may feel a cool sensation in their arm. Initiate the injection. IMPORTANT: Do not begin scanning until the scan delay (determined by the timing bolus procedure) has expired. However, breathing instructions should be initiated when $\sim 5 \mathrm{sec}$ of delay are remaining.

If a power injector is not accessible and one is "hand"-injecting, reattach the saline-filled syringe and flush with $20 \mathrm{ml}$ of saline. The process of switching syringes must be completed as quickly as possible and therefore, it is recommended to use of a three-way stopcock.

22. Obtain a first set of post-contrast images according to Table A15.2.6 as soon as possible. Repeat the same breath-holding procedure as in step 20. Allow the patient $\sim 5 \mathrm{sec}$ to breathe and obtain a second identical set of post-contrast acquisitions.

\section{Sequence 6: Moderately $T_{2}$-weighted FSE with fat suppression}

23. Prescribe the volume to cover the liver. Usually, this will require two separate overlapping breath-holdings.

IMPORTANT: Do not obtain these as interleaved images. The "pause before scan" must be set as "none."

Other than causing lower signal intensity of kidneys and renal collecting structures, the previously administered gadolinium will have little effect on the image, although there may be slightly improved conspicuity of solid liver lesions (Jeong et al., 2001). Other advantages of performing this sequence after contrast agent administration include obtaining the 
important contrast-enhanced images earlier and allowing a longer interval before obtaining the delayed post-contrast images.

24. Instruct the patient to hold his/her breath and run sequence 6 according to Table A15.2.7 (Fig. A15.2.6) with 13 slices. Allow the patient to breathe for 10 seconds, then instruct the patient to hold his/her breath and run the sequence for the additional 13 slices.

\section{Sequence 7: Transverse gradient echo with fat suppression}

$T_{\mathrm{R}}$ should be $\sim 20 \mathrm{msec}$ in this sequence. These images show delayed extracellular space contrast, and all vessels are white because of the combined effects of gadolinium and time

Table A15.2.7 Imaging Parameters for Sequence 6 (Fast Spin Echo)

\begin{tabular}{ll}
\hline Patient position & Supine \\
Scan type & Fast spin echo \\
Imaging plane (orientation) & Transverse \\
Variable bandwidth & Yes \\
Pulse sequence database (PSD) & FRFSE-OPT \\
Central slice or volume center & Center to liver \\
Echo time $\left(T_{\mathrm{E}}\right)$ & $82 \mathrm{msec}$ (effective) \\
Receiver bandwidth $(\mathrm{RBW})$ & $\pm 62 \mathrm{kHz}$ \\
Echo train length $(\mathrm{ETL})$ & 19 \\
Repeat time $\left(T_{\mathrm{R}}\right)$ & $2850 \mathrm{msec}$ \\
Flip angle (FA) & $90^{\circ}$ \\
Fields of view $(\mathrm{FOV}, \mathrm{x}$, FOV $\mathrm{y})$ & $320 \mathrm{~mm}, 240 \mathrm{~mm}$ \\
Resolution $(\Delta x, \Delta y)$ & $1.25 \mathrm{~mm}, 1.67 \mathrm{~mm}$ \\
Number of data points collected $\left(N_{\mathrm{x}}, N_{\mathrm{y}}\right)$ & $256, \sim 144$ \\
Display matrix $\left(D_{\mathrm{x}}, D_{\mathrm{y}}\right)$ & 256,256 \\
Slice thickness $(\Delta z)$ & $7 \mathrm{~mm}$ \\
Number of slices & 26 \\
Slice gap & $0.5 \mathrm{~mm}$ \\
Number of excitations $(\mathrm{NEX})$ & 0.5 (half Fourier) \\
Swap read and phase encoding & No \\
Slice location & Cover the liver \\
Saturation pulses & Superior, inferior \\
Fat suppression & Yes \\
Scan time & 46 sec \\
\hline
\end{tabular}

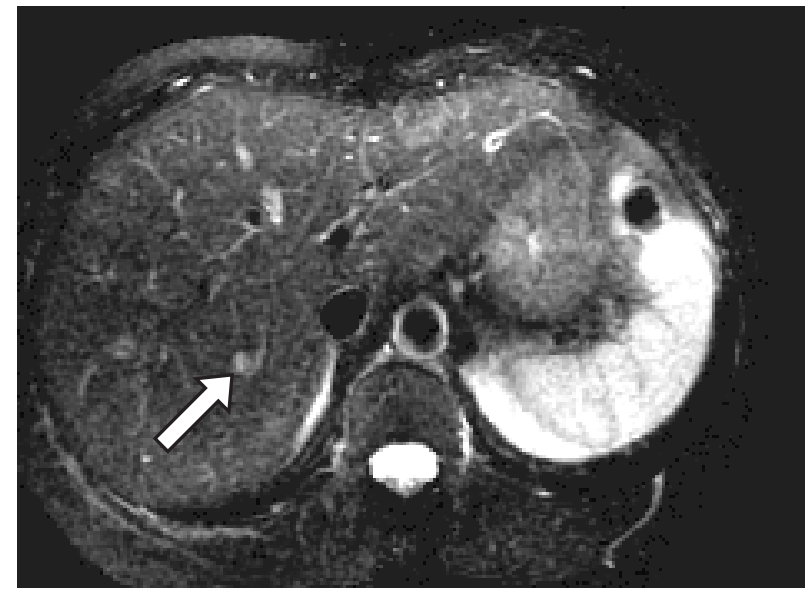

Figure A15.2.6 Moderately $T_{2}$-weighted FSE breath-hold image, with $T_{R}=2850 \mathrm{msec}$, effective $T_{\mathrm{E}}=82 \mathrm{msec}$. Arrow indicates hemangioma. 
of flight (Fig. A15.2.7). Motion artifact is minimal because the short $T_{\mathrm{R}}$ allows completion of each image in $<2$ sec.

25. Instruct the patient to hold his/her breath and run sequence 7 according to Table A15.2.8.

The breath-hold instruction and the way to run this sequence is similar to step 20.

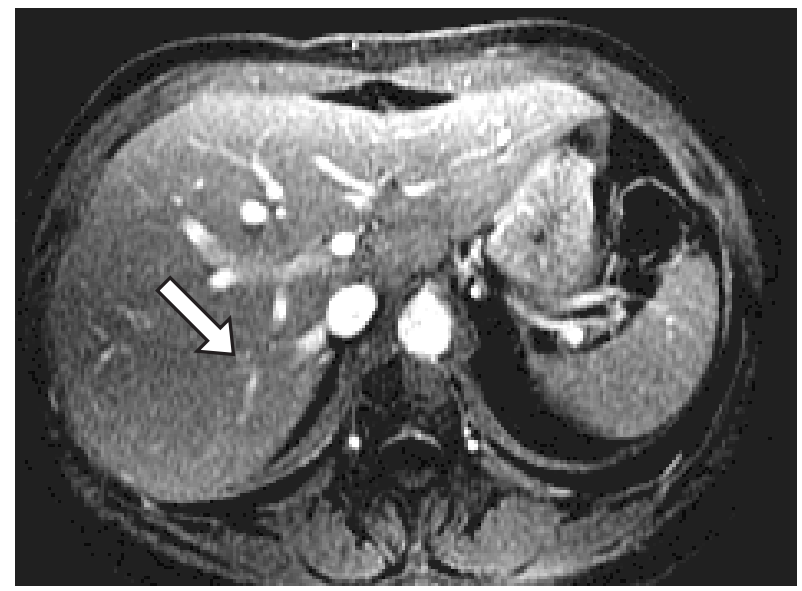

Figure A15.2.7 Two-min post-gadolinium fat-suppressed short $T_{\mathrm{R}}$ gradient echo transverse image (extracellular-phase) demonstrates delayed contrast enhancement, including the cavernous hemangioma (arrow).

Table A15.2.8 Imaging Parameters for Sequence 7 (Fat Suppressed Gradient Echo)

Patient position

Scan type

Imaging plane (orientation)

Variable bandwidth

Pulse sequence database (PSD)

Central slice or volume center

Echo time $\left(T_{\mathrm{E}}\right)$

Receiver bandwidth (RBW)

Repeat time $\left(T_{\mathrm{R}}\right)$

Flip angle (FA)

Fields of view $\left(\mathrm{FOV}_{\mathrm{x}}, \mathrm{FOV}_{\mathrm{y}}\right)$

Resolution $(\Delta x, \Delta y)$

Number of data points collected $\left(N_{\mathrm{x}}, N_{\mathrm{y}}\right)$

Display matrix $\left(D_{\mathrm{x}}, D_{\mathrm{y}}\right)$

Slice thickness $(\Delta z)$

Number of slices

Slice gap

Number of excitations (NEX)

Swap read and phase encoding

Slice location

Fat suppression

Scan time
Supine

2-D gradient echo

Transverse

No

FSPGR

Center to liver

Minimum ( 2.2 msec)

$\pm 16 \mathrm{kHz}$

$20 \mathrm{msec}$

$30^{\circ}$

$320 \mathrm{~mm}, 240 \mathrm{~mm}$

$1.25 \mathrm{~mm}, 2.00 \mathrm{~mm}$

256, 120

256, 256

$5 \mathrm{~mm}$

$\sim 30$

0

1

No

Cover the liver

Yes

$\sim 60 \mathrm{sec}$ 


\section{COMMENTARY}

\section{Background Information}

A comprehensive protocol for liver imaging that includes in-phase and out-of-phase $T_{1^{-}}$ weighted images, moderately and heavily $T_{2^{-}}$ weighted, and early and late post-gadolinium images is described. Each sequence is acquired in one or more breath holds. Total image acquisition time is, therefore, only a few minutes. Most time is spent with patient set-up and with inter-sequence delays. By working efficiently, the entire study can be performed in $\leq 30 \mathrm{~min}$, and will decrease further with user interface enhancements.

At the authors' institution, body MRI studies are obtained from several MRI instruments, including 8 GE Signa scanners at six separate locations. Although referring clinicians are encouraged to include ample clinical information, their compliance with this is highly variable. A set protocol, as described here, ensures that the full spectrum of liver diseases will be detected and characterized without requiring physician intervention. When necessary, attending radiologists can be contacted by phone to give pertinent directions regarding troubleshooting or addressing unusual requests.

\section{Critical Parameters and Troubleshooting}

The two major potential problems with this protocol are that patients may not be able to suspend respiration or timing of gadolinium administration may be incorrect. The first problem can be addressed by replacing sequence 5 with short $T_{\mathrm{R}}(\leq 10 \mathrm{msec})$ single-slice $T_{1^{-}}$ weighted 2-D gradient echo sequences with a flip angle of $\sim 30^{\circ}$. The second problem is prevented in most cases by using a test dose of gadolinium to determine timing (Earls et al., 1997), or by using an automated detection method such as SMARTPREP.

Another problem occurs in patients with implanted metal, such as from surgical clips. Artifacts from metal on gradient echo images can be minimized by using the shortest possible $T_{\mathrm{E}}$ and avoiding fat suppression. $T_{\mathrm{E}}$ on the 3-D short $T_{\mathrm{R}}$ gradient echo sequence is minimized by increasing the number of excitations (NEX) from 0.5 to 1.0, and increasing the sampling bandwidth from \pm 31 to $\pm 62 \mathrm{kHz}$. The acquisition time will be slightly longer, so adjustments in the number of image slices and/or acquisition matrix may be necessary.

\section{Other Contrast Agents}

With good single breath-hold whole-liver dynamic technique, gadolinium chelates are generally considered preferable to other contrast agents for routine liver imaging. Mangafodipir trisodium (Teslascan) or ferumoxides (Feridex) may be considered in some cases, especially those who have already had an equivocal gadolinium-enhanced exam, or to exclude additional tumors prior to curative surgery or ablation.

\section{Anticipated Results}

Most reports comparing MRI and computed tomography (CT) indicate that MRI has higher sensitivity for detecting liver lesions. More importantly, it is more specific for distinguishing benign from malignant lesions. This is partly because the smaller volume of contrast agent and paramagnetic effect of gadolinium contrast agents results in a stronger imaging effect than that of iodinated contrast agents in CT. Additionally, the use of moderately and heavily $T_{2}$-weighted images provides benefits for detecting and characterizing, respectively, focal liver lesions beyond what can be obtained using contrast agents. Heavily $T_{2}$-weighted breathhold images, in particular, allow distinction between benign and malignant lesions in most cases even before the contrast agent is injected (Ito et al., 1997).

\section{Literature Cited}

Earls, J.P., Rofsky, N.M., Lee, V.S., DeCorato, D.R., Krinsky, G.A., and Weinreb, J.C. 1997. Hepatic arterial-phase dynamic gadolinium-enhanced MR imaging: Optimization with a test examination and a power injector. Radiology 202:268273.

Ito, K., Mitchell, D.G., Outwater, E.K., Szklaruk, J., and Sadek, A.G. 1997. Hepatic lesions: Discrimination of nonsolid, benign lesions from solid, malignant lesions with heavily $T_{2}$ weighted fast spin-echo MR imaging. Radiology 204:729-737.

Jeong, Y.Y., Mitchell, D.G., and Holland, G. 2001. Liver lesion conspicuity on $T_{2}$-weighted breathhold fast spin-echo MR imaging before and after gadolinium administration: Initial experience. Radiology 221:117-121.

Marks, B., Mitchell, D.G., and Simelaro, J.P. 1997. Breath-holding in healthy and pulmonary-compromised populations: Effects of hyperventilation and oxygen inspiration. J. Magn. Reson. Imaging 7:595-597.
Liver

A15.2.13

Supplement 4 
Shellock, F.G. and Kanal, E. 1996. Magnetic Resonance Bioeffects, Safety, and Patient Management. Lippincott Williams and Wilkins, Philadelphia.

\section{Key References}

Shellock and Kanal, 1996. See above.

Covers a number of important patient management issues related to MR imaging, including recommended safety procedures, a list of metallic implants that have been tested for MR compatibility, and a list of other sources on MR safety.

\section{Internet Resources}

http://www.mri.tju.edu

A noncommercial site that lists all body MRI protocols, continually updated, updated GE Signa scan- ners by the Thomas Jefferson University Department of Radiology. Additionally, there are descriptions and explanations of the various pulse sequences, tips for problems solving, and examples of clinical applications.

http://www.mrisafety.com

Managed by Frank Shellock, contains updated items regarding MRI safety and compatibility.

Contributed by Donald G. Mitchell,

Peter Natale, and George Holland

Thomas Jefferson University

Philadelphia, Pennsylvania 